\title{
GEF模型对 $n+{ }^{233} \mathrm{U}$ 反应裂变碎片质量分布的研究
}

\author{
郝艺伟 1,2 ，董国香 ${ }^{*}$ ，王小保 ${ }^{2}$ ，王华磊，舒能川 $3^{*} ，$ 陈永静 ${ }^{3}$ ，刘丽乐 ${ }^{3}$ \\ 1. 郑州大学物理工程学院, 郑州 450001; \\ 2. 湖州师范学院理学院, 湖州 313000 ; \\ 3. 中国原子能科学研究院核数据重点实验室, 北京 102413 \\ *联系人, 董国香, E-mail: gxdong@zjhu.edu.cn; 舒能川, E-mail: nshu@ @iae.ac.cn
}

收稿日期: 2019-06-14; 接受日期: 2019-07-30; 网络出版日期: 2019-10-23

国家自然科学基金(编号: 11605054, U1732138, 11505056, 11847315, 11790320, 11790323, 11790325, 11675148)、河南省高等学校青年骨干教师(编号: 2017GGJS008)和郑州大学物理学科推进计划(编号: 32410017)资助项目

\begin{abstract}
摘要 General Fission (GEF)模型是描述裂变过程的一种半经验模型, 它运用了量子力学和统计力学中的 物理概念, 并结合经验信息调整出了一组适用于不同裂变系统的参数, 可以对大量原子核的裂变可观测量 给出可靠的预测. 本工作使用 GEF模型计算了 ${ }^{233} \mathrm{U}$ 中子诱发裂变产额的质量分布. 结果表明, 随着入射中子 能量增加, 对称裂变贡献逐渐增大, 非对称裂变贡献逐渐减小; 考虑了多次机会裂变, (n,f)裂变道相对贡献随 着入射中子能量增加逐渐降低, 而其他裂变道贡献增加; 裂变产物的产额能量变化趋势与其在质量分布上 的不同位置有关.
\end{abstract}

关键词 ${ }^{233} \mathrm{U}, \mathrm{GEF}$ 模型, 裂变产额, 质量分布

PACS: $21.10 . \mathrm{Gv}, 25.85 . \mathrm{Ec}, 24.75 .+\mathrm{i}$

\section{1 引言}

核裂变为核能利用的主要途径, 针作为新一 代核燃料受到人们越来越多的关注, 而中子诱 发 ${ }^{233} \mathrm{U}$ 裂变, 作为关键环节在针铀循环研究中具 有重要的意义 ${ }^{[1]}$. 准确评价裂变过程中的裂变产物 产额对衰变热的计算、核反应堆设计与运行以及乏 燃料处理等方面都具有实际应用意义. 同时, 研究裂 变产额的分布对核裂变机制和核结构研究也具有十 分重要的意义.

核裂变是一个非常复杂的过程, 自从裂变被发
现以来, 人们已经做了无数的尝试来描述裂变过程, 但至今仍遗留许多未解决的问题, 如产额能量关系 的演化. 实验数据对于充分理解裂变过程十分重要, 然而, 短寿命核素的裂变产额数据实验上很难进行 测量, 而且有些实验所测得的产额数据分歧较大, 甚 至在一些能区没有实验数据 ${ }^{[2,3]}$. 在这种情况下, 建 立裂变模型对裂变产额数据进行理论预测是非常必 要的.

沿着不同的研究方向, 物理学家发展了许多不 同的理论模型来描述裂变过程, 如密度泛函理论 框架下采用Skyrme或Gogny有效相互作用的HFB 理

引用格式: 郝艺伟, 董国香, 王小保, 等. GEF模型对 $n+{ }^{233} \mathrm{U}$ 反应裂变碎片质量分布的研究. 中国科学: 物理学 力学 天文学, 2019, 49: 122001 Hao Y W, Dong G X, Wang X B, et al. Mass distributions of fission fragments for $n+{ }^{233} \mathrm{U}$ reaction investigated by GEF model (in Chinese). Sci Sin-Phys Mech Astron, 2019, 49: 122001, doi: 10.1360/SSPMA-2019-0222 
论 ${ }^{[4,5]}$. 这种方法为我们提供了裂变产额的量子力 学微观解释, 但需要进行大量的数值计算, 而且在核 技术应用方面, 这种平均场方法的精确性有待进一 步提高. 在系统学研究的基础上, 也有完全经验的 方法, 如五高斯模型 ${ }^{[6]}$. 在这个模型中, 裂变产物产 额分布是用 5 个高斯分布之和来描述的, 但这样的经 验模型缺乏物理基础, 难以解释裂变过程中的物理 现象. 这两者之间还有一些是用半经验唯象模型, 比 如Benlliure等人 ${ }^{[7]}$ 和 Shu等人 ${ }^{[8]}$ 的工作, 根据实验数 据拟合出模型参数, 能有效再现裂变产额数据, 同时 保留了基本的物理思想, 但该模型拟合计算极大地 依赖实验数据及相应误差, 所以有一定的局限性 ${ }^{[9]}$. Schmidt等人 ${ }^{[10]}$ 提出的GEF唯象模型避免了微观计 算的近似性和局限性, 简化计算的同时又纳入了许 多基本的物理思想. GEF模型参数较多, 一些参数 值是通过拟合大量实验数据确定的, 无法像微观模 型那样能把这些模型参数值与核力的性质联系起 来, 但GEF目前是更加适合应用的程序. 在本文中, $\mathrm{GEF}$ 唯象模型将用于 ${ }^{233} \mathrm{U}$ 中子诱发裂变产额分布规 律的研究.

\section{2 理论模型}

GEF是模拟核裂变过程的计算程序, 是MonteCarlo编码, 它将量子力学和统计力学中的物理概念 与特定的实验信息结合在一起, 采用一种通用的方 法描述所有裂变可观测量. 比如中子诱发裂变瞬发 中子发射前和发射后裂变碎片质量产额、核素产 额、角动量分布、同核异能态产额、瞬发中子谱和 瞬发伽玛谱等. 考虑了多种裂变模式和多次机会裂 变, 在前人的研究基础上, 模型获得参数经验公式和 初始值, 然后通过对更多裂变可观测量的拟合, 获得 最佳参数. 模型虽然也依赖于实验数据, 但普适性 强, 使用范围广泛, 可以自洽地计算所有裂变物理 量, 通过多个计算程序(TALYS, CYFP, GEF)对多个 裂变系统的计算比较, GEF计算结果与大多数实验 数据(包括新获取的实验数据) 符合较好, 被认为具 有良好的预测性. JEFF-更新版 $3.3^{[11]}$ 参考了 GEF计 算结果.

裂变碎片的产额是由裂变垒和断点之间的势能
面的性质决定的, 势能面是质量不对称自由度 的函数. 根据宏观-微观方法, 断点的势能由 液滴模型给出的宏观势和壳效应之和得出 ${ }^{[12]}$. Mosel和Schmitt ${ }^{[13]}$ 的研究发现, 外垒附近的单粒子 能级结构与两个裂变碎片的单粒子能级结构十分相 似. 用量子力学的一般特性来解释这一结果, 即颈 状势(Necked-in Potential) 中波函数的主要部分已经 局域在将要形成的裂变碎片的位置. 这一发现意味 着裂变系统的微观性质本质上是由碎片壳层结构决 定的, 而宏观性质与特定的裂变系统有关 ${ }^{[14]}$. GEF 模型利用这一“可分性原则”(Separability Principle), 通过经验信息来确定宏观势的硬度以及碎片壳的位 置和强度. GEF 模型使用了大约 50 个参数, 从经验 数据的系统分析确定了一些参数, 这些参数经过调 整以适应大量的裂变系统, 即只需要一组参数就可 以给出大量裂变核的结果, 而不需要对于某个特定 裂变系统进行单独调整. 文献[10]中给出了裂变道 的位置和宽度、壳效应和碎片形变等参数及其不确 定性. 由于模型的参数与系统的物理性质密切相关, 因此可以推导出一些关于裂变过程的有趣结论.

在Brosa等人 ${ }^{[15]}$ 提出的多模式无规颈断裂模型 中, 裂变核有多种裂变路径(裂变模式 ${ }^{[16]}$. 一般对 于钶系核主要考虑最重要的 3 种裂变模式, 分别为超 长形变(SL)模式、标准I (S1)模式和标准II (S2)模式, 但在本工作中还考虑了第4种裂变模式, 即超级不对 称(SA)裂变模式 ${ }^{[17,18]}$, 每种裂变模式对应势能曲面 上不同的裂变路径及断前形状, 其中SL是对称裂变, 呈现对称的产额分布, 碎片具有较低的总动能, 且两 个裂变碎片在断点处有强烈形变. 标准 $\mathrm{S} 1$ 和 $\mathrm{S} 2$ 模式 是非对称裂变, 呈现不对称的质量分布, 碎片具有 较高的总动能, 其中 $\mathrm{S} 1$ 模式又比 $\mathrm{S} 2$ 模式高. $\mathrm{S} 1$ 裂变 模式被认为是由一个靠近双幻核 ${ }^{132} \mathrm{Sn}$ 的球形重碎 片和一个中等形变的轻碎片构成. S2模式为一个中 等形变重碎片和中等形变的轻碎片构成. SL模式与 液滴宏观势能相关, S1, S2 模式与重碎片的壳效应 相关. $\mathrm{S} 1$ 重碎片的平均质量在 134 附近, 接近于 $Z=50$, $N=82$ 的球形壳, $\mathrm{S} 2$ 重碎片的平均质量在 140 附近, 靠近 $N=88$ 的形变壳 ${ }^{[9,19]}$.

对于热中子诱发裂变, 各不对称裂变模式电荷 断点的平均位置由以下经验公式给出: 
$\bar{Z}_{S 1}=51.5+25 \cdot\left(\frac{Z_{\mathrm{CN}}^{1.3}}{A_{\mathrm{CN}}}-1.5\right)$,

$\bar{Z}_{S 2}=53.4+21.67 \cdot\left(\frac{Z_{\mathrm{CN}}^{1.3}}{A_{\mathrm{CN}}}-1.5\right)$,

$\bar{Z}_{S A}=58.0+21.67 \cdot\left(\frac{Z_{\mathrm{CN}}^{1.3}}{A_{\mathrm{CN}}}-1.5\right)$,

其中 $A_{\mathrm{CN}}$ 是复合核的质量数, $Z_{\mathrm{CN}}$ 是复合核的质子数. 碎片壳的位置被参数化为核电荷 $Z$ 的函数, 在不同 的裂变系统中, 各裂变道电荷平均断点位置是基本 不变的, 而碎片质量断点位置则会随激发能量的增 加而发生变化.

裂变产额由下式计算:

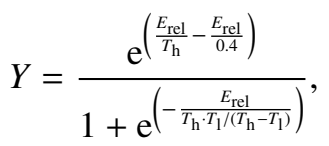

其中 $Y$ 代表通过某个裂变途径的裂变几率, $E_{\mathrm{rel}}$ 为相 对于位垒的能量, $T_{\mathrm{h}}$ 为低于位垒的有效温度, $T_{1}$ 为高 于位垒的有效温度. 各个裂变模式的相对产额由下 式给出:

$Y_{2} / Y_{1}=\mathrm{e}^{-\Delta E / T} \cdot \frac{\hbar \omega_{1}}{\hbar \omega_{2}} \approx \mathrm{e}^{-\Delta E / T}$,

其中 $Y_{1}, Y_{2}$ 代表两个不同裂变途径的裂变几率, $\Delta E$ 是两个裂变位垒差. 当激发能小于裂变位垒 时, 考虑位垒穿透之后的裂变几率公式由下式给出:

$Y_{i}=\frac{\mathrm{e}^{E_{B, i}^{*} / T}}{1+\mathrm{e}^{\left(-E_{B, i}^{*} /\left(T \cdot T_{\text {tum }}^{i} /\left(T-T_{\text {tun }}^{i}\right)\right)\right.}}$,

其中 $E_{B, i}^{*}$ 为相对于裂变位垒的能量, 等于裂变核的初 始激发能减去外垒高度, $E_{B, i}^{*}$ 对于各裂变道相对产额 的影响用斜率参数为 $T_{\text {tun }}^{i}$ 的指数函数可以很好地描 述. 基于文献[20]的能级密度参数化给出由宏观势 形成的对称裂变道的温度参数, 它在等温条件下具 有的最小值为 $0.72 \mathrm{MeV}$. 质量不对称自由度下的集 体温度 $T$ 由下式给出:

$T=0.034 \cdot E_{B}^{*}+0.04 \cdot \Delta E_{\mathrm{pot}}$,

其中 $\Delta E_{\mathrm{pot}}$ 为从鞍点到断点过程中增加的势能,

$\Delta E_{\mathrm{pot}}(\mathrm{MeV})=0.08 \cdot\left(Z_{\mathrm{CN}}^{2} / A_{\mathrm{CN}}^{1 / 3}-1358\right)+11$,
裂变位垒计算时考虑了对能和壳修正, 但在鞍点的 壳效应比较小可以忽略 ${ }^{[21]}$, 所以裂变位垒由以下三 部分构成: 宏观裂变位垒减去基态微观贡献加上垒 上微观贡献:

$B_{\mathrm{f}}=B_{\mathrm{f}}^{T F}-\delta E_{\mathrm{gs}}^{\mathrm{mic}}+\delta E_{\mathrm{f}}^{\mathrm{mic}}$,

其中基态微观贡献为 $\delta E_{\mathrm{gs}}^{\mathrm{mic}}=m_{\mathrm{gs}}-m_{T F}$, 垒上微观贡 献为 $\delta E_{\mathrm{f}}^{\text {mic }}=\delta P_{\mathrm{f}}$, 最终得到

$B_{\mathrm{f}}=B_{\mathrm{f}}^{T F}-\left(m_{\mathrm{gs}}-m_{T F}\right)+\delta P_{\mathrm{f}}$.

裂变核在断点的激发能由三部分构成:

$E_{\text {scission }}^{*}=E_{B}^{*}+E_{\text {diss }}+E_{\text {coll }}$,

$E_{\text {diss }}=0.35 \cdot \Delta E_{\mathrm{pot}}$,

$E_{\text {coll }}=0.065 \cdot \Delta E_{\text {pot }}^{\text {coll }}$,

$\Delta E_{\mathrm{pot}}^{\mathrm{coll}} / \mathrm{MeV}=0.08 \cdot\left(Z_{\mathrm{CN}}^{2} / A_{\mathrm{CN}}^{1 / 3}-1390\right)+11$,

其中 $E_{\text {diss }}$ 为在从鞍点到断点过程中获得的内禀能, 约为鞍点-断点势能差的 $35 \%, E_{\text {coll }}$ 为集体自由度 之间耦合获得的集体能, 约为鞍点-断点势能差 的 $6.5 \%$. 对于某一裂变核的多次机会裂变各裂变 道的激发能不同.

\section{3 结果与讨论}

用GEF程序和系统学计算程序 CYFP ${ }^{1)}$ 计算了 中子入射能量为热能点和 $14.8 \mathrm{MeV}$ 产额质量分布. GEF计算结果与CYFP计算结果以及EXFOR数据库 实验数据相比较如图1和 2 所示, CYFP程序在入射中 子能量为 $14.8 \mathrm{MeV}$ 时与实验数据符合得较好, 而在 热能点时两个峰区产额数值与实验数据相差较大, GEF程序则在两个能点都与实验数据符合较好.

不同的裂变模式对应不同的产额质量分布, 对 于某个裂变核, 其产额质量分布为多种模式的叠 加, 每个模式所占的份额与入射中子能量 $E_{n}$ 有关. 图3为用GEF模型计算的不同裂变模式的相对贡献 随入射中子能量的变化关系. 从图中可看出, S2模 式在0-20 MeV这个能量范围内是占优势的, 随着入

1) Wahl A C. Systematics of Fission-Product Yields. Los Alamos National Laboratory Report. No. LA-13928, 2002 (unpublished) 
射中子能量升高, 壳效应逐渐减弱, 非对称裂变 $\mathrm{S} 1$, $\mathrm{S} 2$ 模式贡献逐渐减小. SA模式所占贡献非常小, 从 热能点到 $2 \mathrm{MeV}$ 左右贡献很小, 总体变化趋势是随 中子能量增加贡献减小. 对称裂变SL模式贡献逐渐 增加, 非对称裂变模式逐渐转化为对称裂变模式.

GEF模型可以计算各裂变模式和各裂变道相应 的分质量分布. 图4给出了SL, S1, S2, SA四种裂变模 式在不同入射中子能量下各自的产额质量分布, 可 明显看出非对称裂变 $S 1, S 2$ 均近似为双峰分布, 且 随入射中子能量的升高峰高降低峰宽增大, 壳效应 逐渐减弱, 贡献减少. 为了便于观察把它转换到对 数坐标上, 可以看出对称裂变为单峰分布, 随着能量

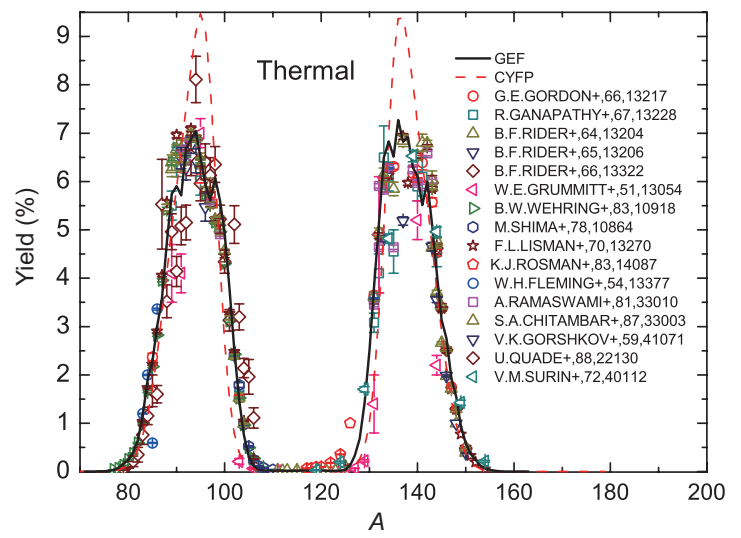

图 1 (网络版彩图)热能点 $\mathrm{n}+{ }^{233} \mathrm{U}$ 裂变碎片质量分布

Figure 1 (Color online) Mass distributions of fission fragments of $\mathrm{n}+{ }^{233} \mathrm{U}$ reaction at $E_{n}=$ Thermal.

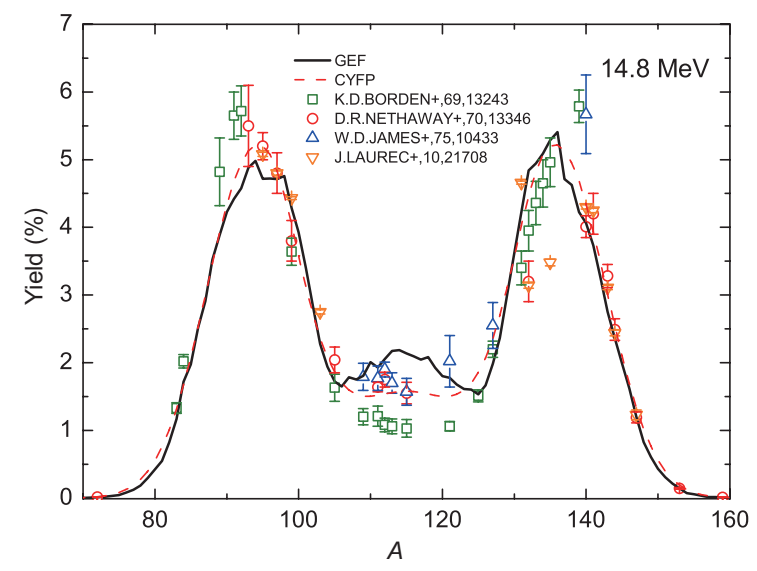

图 2 (网络版彩图) $14.8 \mathrm{MeV} \mathrm{n}+{ }^{233} \mathrm{U}$ 裂变碎片质量分布

Figure 2 (Color online) Mass distributions of fission fragments of $\mathrm{n}+{ }^{233} \mathrm{U}$ reaction at $E_{n}=14.8 \mathrm{MeV}$.

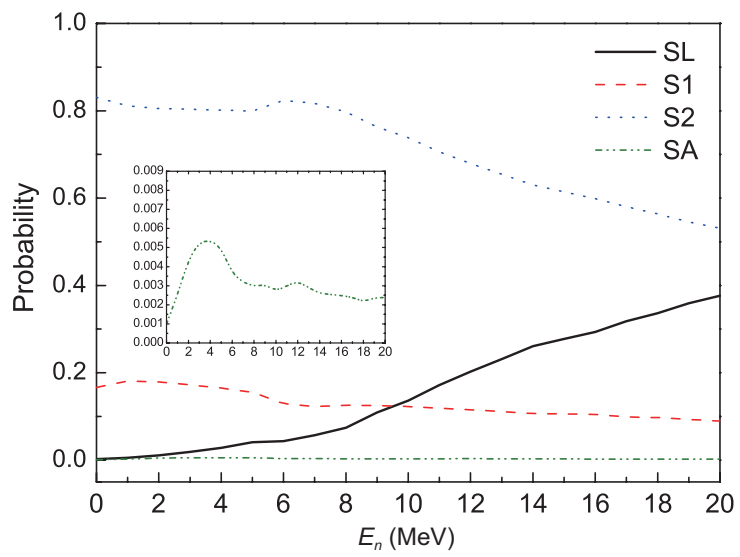

图 3 (网络版彩图)不同裂变模式的相对贡献随入射中子能 量的变化关系

Figure 3 (Color online) The relative contribution of different fission modes as a function of the incident neutron energy.

的升高, 峰高与峰宽均增大, 贡献增加. 超级不对 称模式SA是比较特殊的, 可以从图中看出SA模式 为双峰分布, 虽然SA 模式在热能点的贡献比在 $14 \mathrm{MeV}$ 时的贡献小, 但由图3可知, 其随入射中子 能量变化的总体趋势是随能量升高贡献减小. 而 且SA模式所占的份额非常小, 几乎可以忽略不计. 此外 $\mathrm{S} 1$ 和 $\mathrm{S} 2$ 模式的位置几乎不变, 这也与壳效应相 对应.

随着入射中子的能量增加, 复合核在裂变之前 可能会先发射一个或几个中子, 发射中子后, 还有足 够的激发能发生裂变, 即多次机会裂变, (n,f), (n,nf), $(\mathrm{n}, 2 \mathrm{nf})$ 等多道裂变道会打开. 图5为计算的多次机会 裂变不同裂变道的相对贡献随入射中子能量的变 化关系. 从图中可以看出, 入射中子能量 $E_{n}<20 \mathrm{MeV}$ 时主要有三个裂变道, 能量较低时只有 $(n, f)$ 裂变道, $E_{n}$ 到 $5 \mathrm{MeV}$ 左右时 $(\mathrm{n}, \mathrm{nf})$ 裂变道打开, $E_{n}$ 到 $11 \mathrm{MeV}$ 左 右时 $(\mathrm{n}, 2 \mathrm{nf})$ 裂变道也打开. 随入射中子能量升高, $(\mathrm{n}, \mathrm{f})$ 裂变道相对贡献逐渐降低, 其他裂变道的贡献 逐渐增加. 某一裂变系统下, 总的产额质量分布为 各个裂变道的权重和.

图6给出了 $(\mathrm{n}, \mathrm{f}),(\mathrm{n}, \mathrm{nf}),(\mathrm{n}, 2 \mathrm{nf})$ 三个裂变道在 $E_{n}=6 \mathrm{MeV}$ 和 $14 \mathrm{MeV}$ 时的产额质量分布. 在 $E_{n}=$ $6 \mathrm{MeV}$ 时主要有两个裂变反应道, 峰区和谷区都是 $(\mathrm{n}, \mathrm{f})$ 裂变道占主要优势. 当 $E_{n}=14 \mathrm{MeV}$ 时主要有 3 个 裂变道, 谷区 $(n, f)$ 裂变道占优势, 峰区则是 $(n, n f)$ 裂变 

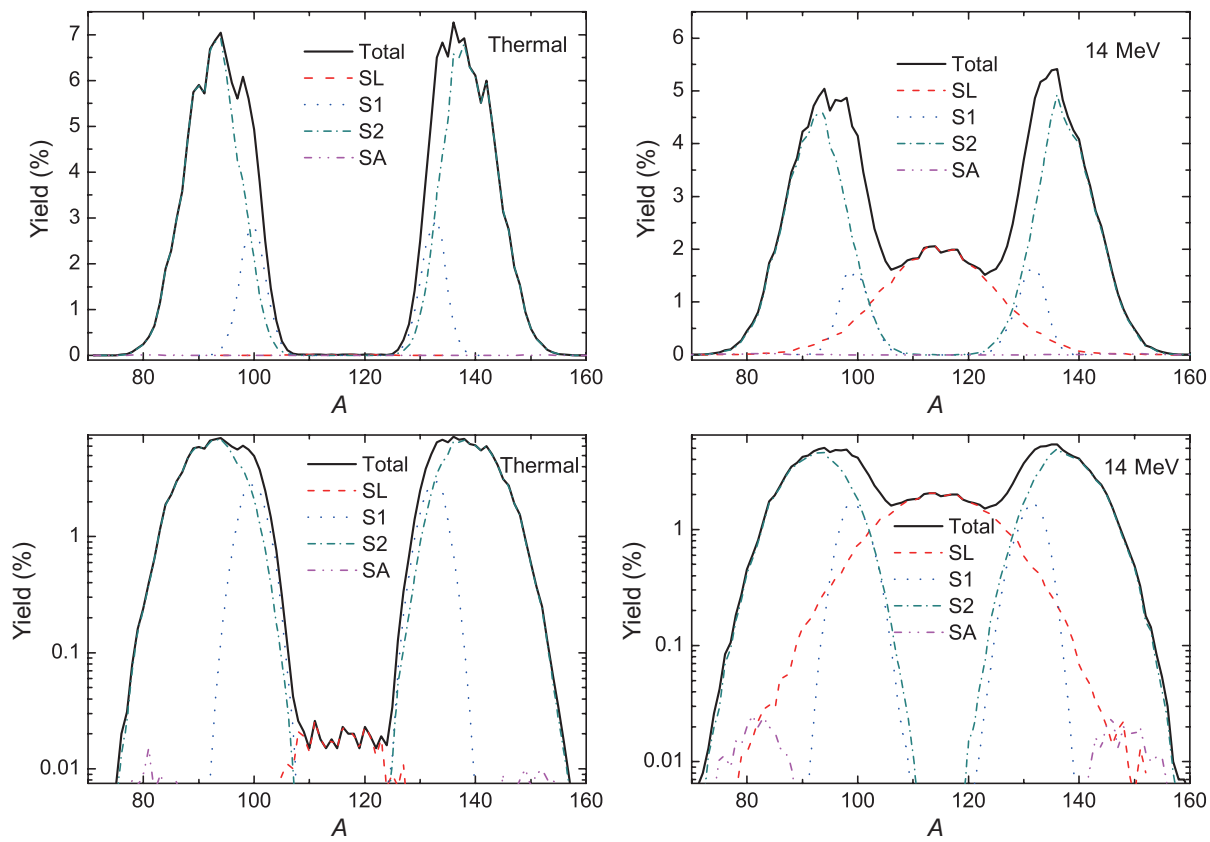

图 4 (网络版彩图) 不同裂变模式的分质量分布, 上为直角坐标, 下为对数坐标, 左边 $E_{n}=\mathrm{Thermal}$, 右边 $E_{n}=14 \mathrm{MeV}$

Figure 4 (Color online) Calculated mass distributions of different fission modes at $E_{n}=$ Thermal and $14 \mathrm{MeV}$. The same results are plotted in linear and logarithmic scales, respectively, in the upper and lower panels.

道占优势.

裂变碎片产额分布与入射中子的能量有关, 且质量分布上不同位置的产物核产额能量关系 明显不同. 图7为计算的入射中子能量从热能点到 $20 \mathrm{MeV}$ 共6个能点的产额质量分布, 可发现随着入 射中子能量的增加产额质量分布的变化有如下特 点: 随着能量的升高, 壳效应逐渐减弱, 非对称裂变

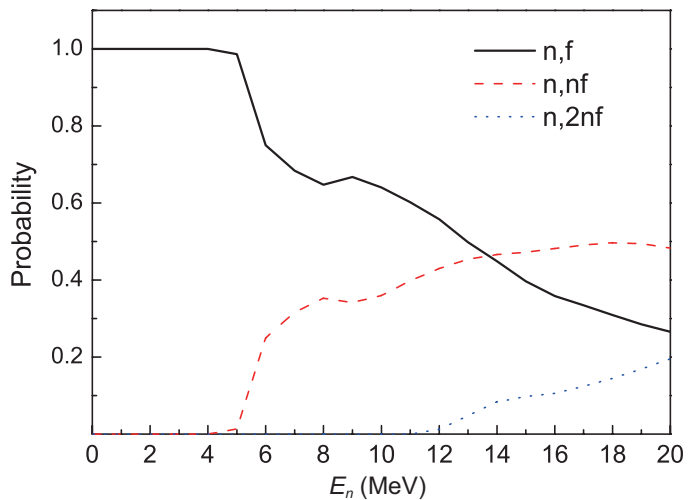

图 5 (网络版彩图)多次机会裂变不同裂变道的相对贡献随 入射中子能量的变化关系

Figure 5 (Color online) The relative contribution of different fission chances as a function of the incident neutron energy.
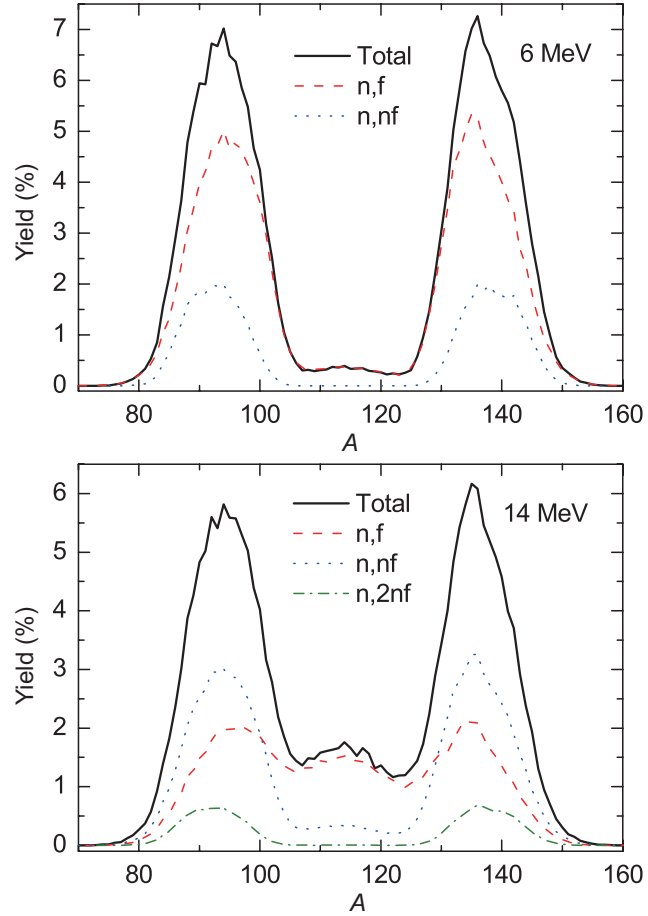

图 6 (网络版彩图)多次机会裂变不同裂变道的分质量分布, 上为 $E_{n}=6 \mathrm{MeV}$, 下为 $E_{n}=14 \mathrm{MeV}$

Figure 6 (Color online) Calculated mass distributions of the different fission chances at $E_{n}=6 \mathrm{MeV}$ and $14 \mathrm{MeV}$. 


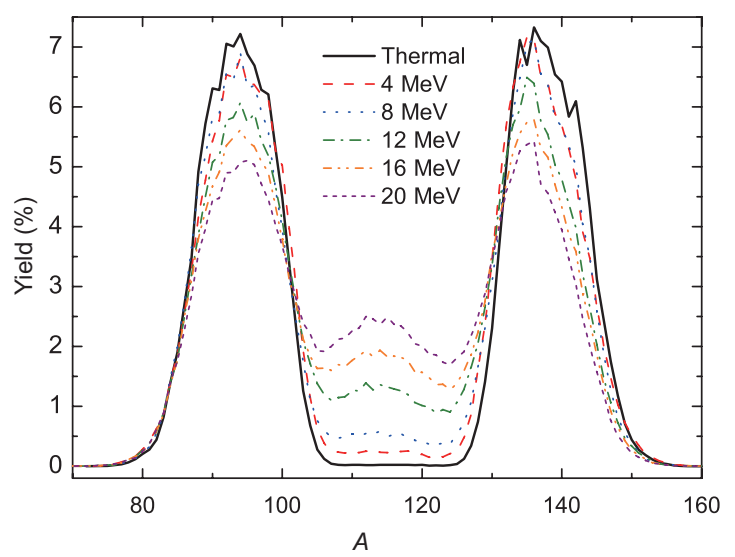

图 7 (网络版彩图)不同能点的质量产额分布

Figure 7 (Color online) Calculated mass distributions at different incident neutron energies.

的贡献减小, 对称裂变贡献增加, 峰高变低, 谷底 抬高, 峰宽加大. 质量分布不同位置上产额随能量 变化会出现不同的趋势: 轻峰区 $(A \sim 85-101)$ 和重峰 区 $(A \sim 132-148)$ 产额总的趋势随入射中子能量升高 而下降, 谷区和两翼产额随入射中子能量上升而上 升. 上述 4 个质量点称为趋势转折点, 外侧两个点、 内侧两个点之和, 考虑中子发射带走的 2-3个中子之 后, 与复合核的质量几乎相等. 这与刘丽乐等人 ${ }^{[9]}$ 给 出结果一致.

\section{4 总结}

裂变的半经验模型GEF模型采用一种全局方法 使用一组参数可以计算大量原子核的裂变可观测 量, 而不需要各个裂变系统具体的实验信息, 可以为 产额评价提供重要的参考. 本工作使用 GEF模型计 算 ${ }^{233} \mathrm{U}$ 中子诱发裂变各裂变模式和多次机会裂变各 个裂变道的产额质量分布并对产能关系进行了研 究. 计算结果表明, 随着入射中子能量的提高, 壳效 应减弱, 对称裂变贡献逐渐增大, 非对称裂变贡献逐 渐减小. 入射中子能量增加到一定值时多次机会裂 变各裂变道会依次打开, 随着能量增高 $(\mathrm{n}, \mathrm{f})$ 裂变道 相对贡献逐渐降低, 其他裂变道贡献增加. 质量分 布不同位置上产额随能量变化有不同的变化趋势, 峰区产额随入射中子能量升高而下降, 谷区产额随 入射中子能量上升而上升.

通过GEF, CYFP计算结果和实验数据的比较, 说明CYFP程序是系统学程序, 并且自2002年以来没 有更新, 峰区数据与实验数据相差较大; 而GEF这些 年来一直在更新, 与现有的实验数据或者评价数据 符合较好, 为缺乏产额实验数据的核提供了较为可 靠的预测, 因此推荐GEF作为评价数据的参考. 在下 一步工作中, 我们将对美国利弗莫尔国家实验室开 发的FREYA 程序 ${ }^{[22]}$ 进行解读.

\section{参考文献}

1 IAEA N F C. Thorium Fuel Cycle - Potential Benefits and Challenges-IAEA-TECDOC-1450. Technical Report, Vienna: International Atomic Energy Agency. 2005

2 Böckstiegel C, Steinhäuser S, Schmidt K H, et al. Nuclear-fission studies with relativistic secondary beams: Analysis of fission channels. Nucl Phys A, 2008, 802: 12-25

3 Kellett M A, Besillon O, Mills R W. The JEFF-3.1/-3.1.1 Radioactive Decay Data and Fission Yields Sub-libraries. Technical Report, No. 6287, OECD Nuclear Energy Agency. 2009

4 Schunck N, Duke D, Carr H, et al. Description of induced nuclear fission with Skyrme energy functionals: Static potential energy surfaces and fission fragment properties. Phys Rev C, 2014, 90: 054305

5 Regnier D, Dubray N, Schunck N, et al. Fission fragment charge and mass distributions in ${ }^{239} \mathrm{Pu}(\mathrm{n}, \mathrm{f})$ in the adiabatic nuclear energy density functional theory. Phys Rev C, 2016, 93: 054611

6 Musgrove A R de L, Cock J L, Trimble G D. Prediction of Unmeasured Fission Product Yields. IAEA-169. Technical Report, Vienna: International Atomic Energy Agency, 1974. 163-200

7 Benlliure J, Grewe A, de Jong M, et al. Calculated nuclide production yields in relativistic collisions of fissile nuclei. Nucl Phys A, 1998, 628: 458-478

8 Shu N C, Chen Y J, Liu T J, et al. Semi-empirical study on the yield energy-dependence of the 235U + n fission. J Korean Phy Soc, 2011, 59: 1353-1356 
9 Liu L L, Shu N C, Liu T J, et al. Study on mass distribution of $\mathrm{n}+{ }^{233} \mathrm{U}$ fission with phenomenological model (in Chinese). Nucl Phys Rev, 2013, 30: 374 [刘丽乐, 舒能川, 刘廷进, 等. $\mathrm{n}+{ }^{233} \mathrm{U}$ 裂变碎片质量分布的唯象模型研究. 原子核物理评论, 2013, 30: 374]

10 Schmidt K H, Jurado B, Amouroux C, et al. General description of fission observables: GEF model code. Nucl Data Sheets, 2016, 131: 107-221

11 Kellett M A, Bersillon O. The Decay Data Evaluation Project (DDEP) and the JEFF-3.3 radioactive decay data library: Combining international collaborative efforts on evaluated decay data. EPJ Web Conf, 2017, 146: 02009

12 Brack M, Damgaard J, Jensen A S, et al. Funny hills: The shell-correction approach to nuclear shell effects and its applications to the fission process. Rev Mod Phys, 1972, 44: 320-405

13 Mosel U, Schmitt H W. Potential energy surfaces for heavy nuclei in the two-center model. Nucl Phys A, 1971, 165: 73-96

14 Schmidt K H, Kelić A, Ricciardi M V. Experimental evidence for the separability of compound-nucleus and fragment properties in fission. Europhys Lett, 2008, 83: 32001

15 Brosa U, Grossmann S, Müller A. Nuclear scission. Phys Rep, 1990, 197: 167-262

16 Fan T, Hu J, Bao S. Study of multichannel theory for the neutron induced fissions of actinide nuclei. Nucl Phys A, 1995, 591: 161-181

17 Mulgin S I, Okolovich V N, Zhdanova S V. Observation of new channel in the proton-induced low-energy fission of nuclei from 233 Pa to 245 Bk. Phys Lett B, 1999, 462: 29-33

18 Hambsch F -J, Oberstedt S, Aarle J Van. ${ }^{252} \mathrm{Cf}(\mathrm{SF})$ : Fission modes and far asymmetric mass yields. Conf Proc-Italian Phys Soc, 1997, 59: 1239

19 Wilkins B D, Steinberg E P, Chasman R R. Scission-point model of nuclear fission based on deformed-shell effects. Phys Rev C, 1976, 14: 1832-1863

20 Rusanov A Ya, Itkis M G, Oklovich V N. Features of mass distributions of hot rotating nuclei. Phys Atom Nucl, 1997, 60: 683

21 Myers W D, Swiatecki W J. Nuclear properties according to the Thomas-Fermi model. Nucl Phys A, 1996, 601: 141-167

22 Verbeke J M, Randrup J, Vogt R. Fission reaction event yield algorithm FREYA 2.0.2. Comput Phys Commun, 2018, 222: 263-266

\title{
Mass distributions of fission fragments for $n+{ }^{233} \mathrm{U}$ reaction investigated by GEF model
}

\author{
HAO YiWei ${ }^{1,2}$, DONG GuoXiang ${ }^{2 *}$, WANG XiaoBao ${ }^{2}$, WANG HuaLei ${ }^{1}$, SHU NengChuan $^{3 *}$, \\ CHEN YongJing ${ }^{3} \&$ LIU LiLe ${ }^{3}$ \\ ${ }^{1}$ School of Physics and Engineering, Zhengzhou University, Zhengzhou 450001, China; \\ ${ }^{2}$ School of Science, Huzhou University, Huzhou 313000, China; \\ ${ }^{3}$ Key Laboratory of Science and Technology on Nuclear Data, China Institute of Atomic Energy, Beijing 102413, China
}

The General Fission (GEF) model is a semi-empirical model describing fission process, which combines physical concepts from quantum mechanics and statistical mechanics with specific experimental information to adjust a set of parameters suitable for different fission systems and gives reliable predictions for essentially all fission observables of a broad range of fissioning nuclei. In this work, the mass distributions of ${ }^{233} \mathrm{U}$ neutron-induced fission are calculated using the GEF model. The results show that with the increase of incident neutron energy, the contribution of symmetric fission channel increases gradually, and the asymmetric fission channel decreases. Detailed analysis of the fragment mass distribution is performed by taking multi-chance fission into account. The contribution of $(\mathrm{n}, \mathrm{f})$ fission channel gradually decreases with the increase of incident neutron energy, while the other fission channels increases. The variation trend of the yield energy-dependence is related to the position located on the mass distribution.

\section{${ }^{233} \mathrm{U}$, GEF model, fission yield, mass distribution}

PACS: $21.10 . \mathrm{Gv}, 25.85 . \mathrm{Ec}, 24.75 .+\mathrm{i}$

doi: $10.1360 /$ SSPMA-2019-0222 